\title{
Fulminant Giant Cell Myocarditis and Cardiogenic Shock: An Unusual Presentation of Malignant Thymoma
}

\author{
Deepak Koul, ${ }^{1}$ Manreet Kanwar, ${ }^{1}$ Dane Jefic, ${ }^{1}$ Anuradha Kolluru, ${ }^{2}$ Tejwant Singh, ${ }^{2}$ \\ Sunil Dhar, ${ }^{3}$ Preetham Kumar, ${ }^{2}$ and Gerald Cohen ${ }^{2}$
}
${ }^{1}$ Division of Cardiology, Cardiac Cath Laboratory, St. John Hospital and Medical Center, 22101 Moross Road, 2nd Floor VEP, Detroit, MI 48236, USA
${ }^{2}$ St. John Hospital and Medical Center, 22101 Moross Road, Detroit, MI 48236, USA
${ }^{3}$ Abington Memorial Hospital, Drexel University College of Medicine, 1200 Old York Raod, Abington, PA 19001, USA

Correspondence should be addressed to Deepak Koul, deekoul@yahoo.com

Received 13 October 2009; Accepted 4 March 2010

Academic Editor: Jalal K. Ghali

Copyright ( $\odot 2010$ Deepak Koul et al. This is an open access article distributed under the Creative Commons Attribution License, which permits unrestricted use, distribution, and reproduction in any medium, provided the original work is properly cited.

Malignant thymoma is rarely associated with giant cell myocarditis. We present a case study that illustrates this association and cardiogenic shock with underlying tamponade. The dramatic presentation of this scenario has not been previously described.

\section{Introduction}

Giant cell myocarditis (GCM) is a rare and frequently fatal type of myocarditis. Patients usually die of heart failure and ventricular arrhythmia unless cardiac transplantation is performed [1]. In addition to the idiopathic or primary form, GCM has been associated with other conditions including autoimmune diseases, granulomatosis, and neoplastic conditions [2]. However, the etiology and pathogenesis of GCM is not well known.

Very few cases of malignant thymoma with GCM have been reported. We describe a case of GCM with dramatic clinical presentation of cardiac tamponade and cardiogenic shock.

\section{Case Report}

A previously healthy 55-year-old woman presented with one-week history of worsening muscle weakness, diplopia, and exertional dyspnea. Physical examination revealed a pale thin female with tachycardia (107 beats/minute), tachypnea (22 breaths/minute), hypotension (blood pressure $86 / 50 \mathrm{mmHg}$ ), jugular venous distension with rapid " $X$ " descent, and distant heart sounds. While the patient was being evaluated in the emergency room, she suddenly had a cardiopulmonary arrest. After resuscitation, an echocardiogram revealed severe left ventricular (LV) dysfunction with cardiac tamponade. Emergent pericardiocentesis was done and $50 \mathrm{~mL}$ of pericardial fluid was drained. An extrapericardial mass compressing the left atrium was also suspected, but images were limited. Electrocardiogram showed $1 \mathrm{~mm}$ ST segment elevation in anterior chest leads (Figure 1). Initial labs revealed elevated troponin I $(8.38 \mathrm{ng} / \mathrm{mL})$ and creatine kinase-MB $(21 \mathrm{ng} / \mathrm{mL})$. Since this suggested possible acute myocardial infarction, she was emergently taken for cardiac catheterization, but no coronary artery disease was detected. She was hemodynamically sustained with inotropes and an intraaortic balloon pump (IABP).

Transesophageal echocardiogram showed global LV severe hypokinesis with ejection fraction $(\mathrm{EF})$ of 10 percent and a large mass indenting the left atrium and pulmonary veins (Figures 2(a) and 2(b)). On CT scan, a large superior mediastinal mass extended to the aortopulmonary window and abutted the aortic arch and pulmonary artery (Figure 3 ). CT guided biopsy revealed thymoma with undifferentiated cells.

Further laboratory evaluation showed elevated acetylcholine receptor antibody, antisarcolemma, and antimyosin antibody titers. Antibody titers of viral etiologies for myocarditis were negative. Steroids and plasmapheresis for thymoma-associated myasthenia gravis resulted in neurological improvement. Patient was taken off of inotropes and IABP. A repeat echocardiogram several days later showed improved 


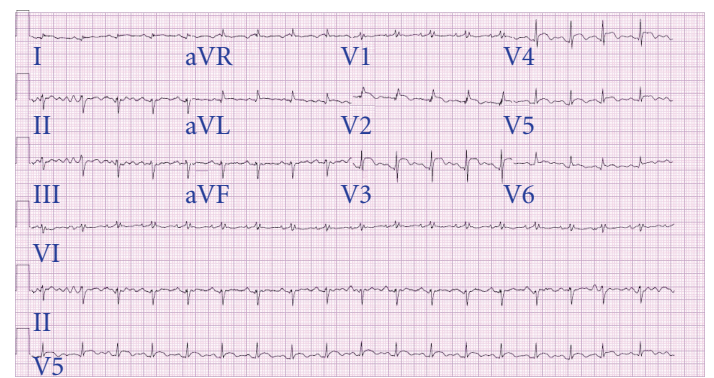

Figure 1: Electrocardiogram showing ST segment elevation in leads I, aVL, and V2-V6.

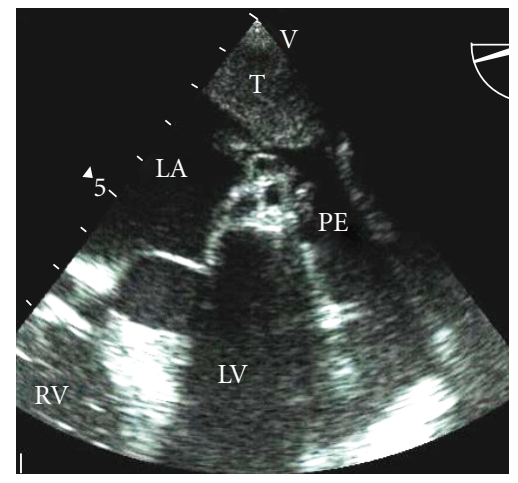

(a)

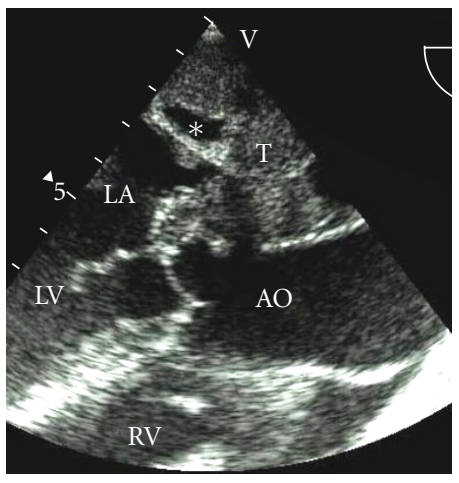

(b)

Figure 2: (a) Transesophageal echocardiogram of 4-chamber view showing tumor mass (T) compressing the left atrium (LA). A pericardial effusion (PE) is adjacent to the left ventricular (LV) free wall. RV = right ventricle. (b) Transesophageal echocardiogram of left ventricular $(\mathrm{LV})$ outflow tract in long axis showing homogenous tumor $(\mathrm{T})$ mass with lucent spaces $(*)$ compressing the left atrium $(\mathrm{LA})$. AO = Ascending aorta. RV = right ventricle.

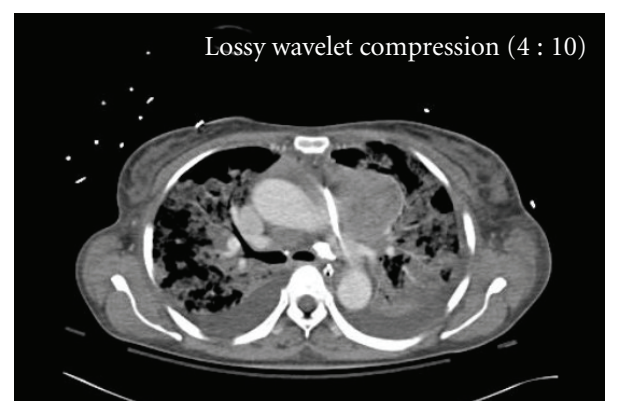

Figure 3: Computed axial tomogram showing a mass in left anterior mediastinum at the root of pulmonary artery with right anterior pericardial involvement and effusion.

LV function with an EF of 45\% (Figure 4). Chemotherapy with carboplatin and paclitaxel resulted in good initial response with CT scan showing reduction in thymoma tumor burden. However, hospital course was complicated by febrile neutropenia and sepsis. She died 35 days after hospital admission.

On autopsy, the heart weighed 439 grams and was uniformly soft and pale without coronary obstruction or valvular abnormality. Histological examination of the myocardium showed large multinucleated epitheliod cells, plasma cells, and lymphocytes characteristic of GCM (Figure 5).

\section{Discussion}

GCM has a rare association with malignant thymoma with or without clinical manifestations of myasthenia gravis [24]. Pathogenesis is unknown and prognosis is very poor. In an animal model, a disorder similar to GCM was induced by immunization with cardiac myosin [5]. Myasthenia gravis is an autoimmune disorder and is found to be associated with thymoma in about $15 \%$ of the cases. Association of giant cell myocarditis with thymoma, myasthenia gravis, and other autoimmune disorders suggests that it may be another manifestation of "immune dysregulation". In this 


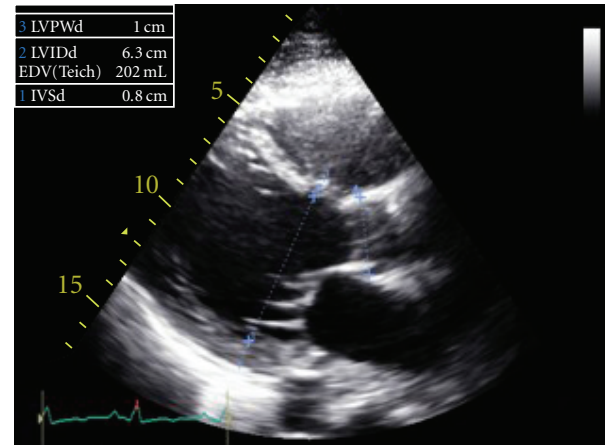

(a)

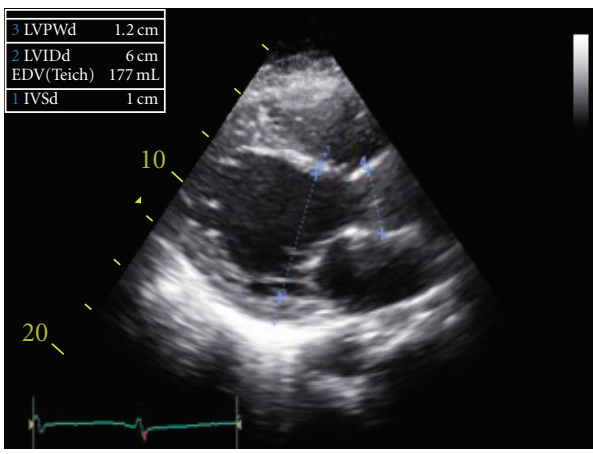

(c)

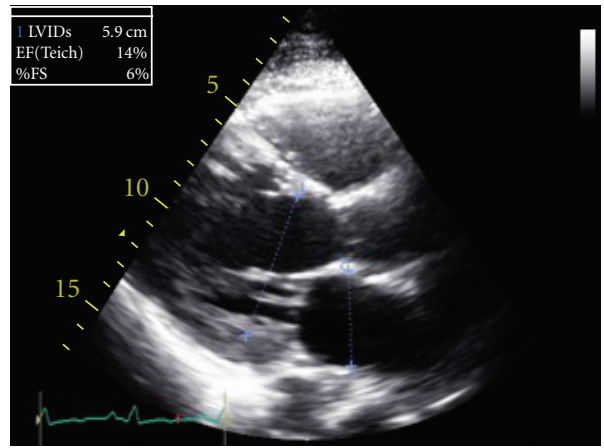

(b)

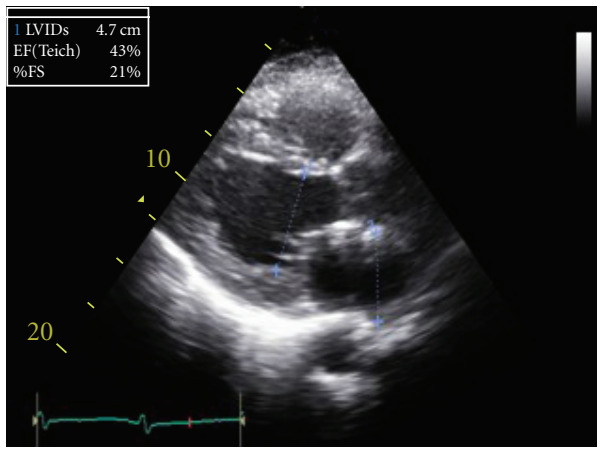

(d)

FIGURE 4: Parasternal long-axis views showing severe baseline LV dysfunction (Figures 4(a) and 4(b)) and improved ejection fraction on the followup study (Figures 4(c) and 4(d)).

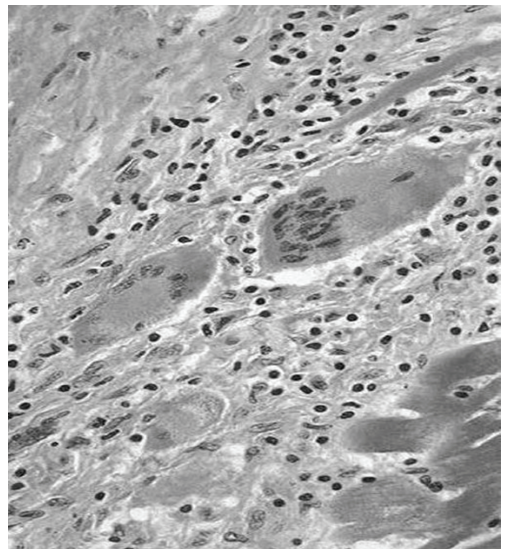

FIGURE 5: Histopathology demonstrating multinucleated giant cells and numerous lymphocytes infiltrating the cardiomyocytes.

case report, myasthenia gravis was confirmed by finding high titers of acetylcholine receptor antibodies. Other nonspecific antibodies like antisarcolemma and antimyosin antibodies provided suggestive evidence that GCM is an autoimmune disease of the myocardium.

Presentation and clinical course of GCM is usually characterized by rapid deterioration in LV function, frequent ventricular arrhythmias, and heart block [1]. In our patient, cardiac tamponade and cardiogenic shock was secondary to underlying GCM. Our patient had fulminant presentation; however, no significant ventricular arrhythmia was seen.

Unlike other types of myocarditis, GCM has been shown to respond to immunosuppressive therapy with significant improvement in survival $[1,6]$. Improvement in LV function in our patient may have been due to immunosuppressive drug treatment, though she died because of sepsis. Autopsy confirmed GCM and its rare association with malignant thymoma.

\section{Conclusion}

This case illustrates the unusual association of thymoma and myasthenia gravis with fulminant myocarditis. To our knowledge, thymoma-associated GCM presenting as cardiac tamponade and cardiogenic shock has not been reported. Myocarditis was likely due to autoantibodies. Immunosuppressive therapy was associated with improved LV function, but ultimately fatal sepsis. This case also highlights the systemic nature of malignant thymoma.

\section{References}

[1] L. T. Cooper Jr., G. J. Berry, and R. Shabetai, "Idiopathic giantcell myocarditis-natural history and treatment. Multicenter Giant Cell Myocarditis Study Group Investigators," New England Journal of Medicine, vol. 336, no. 26, pp. 1860-1866, 1997. 
[2] C. M. Kilgallen, E. Jackson, M. Bankoff, R. N. Salomon, and H. K. Surks, "A case of giant cell myocarditis and malignant thymoma: a postmortem diagnosis by needle biopsy," Clinical Cardiology, vol. 21, no. 1, pp. 48-51, 1998.

[3] T. M. Joudinaud, E. Fadel, V. Thomas-de-Montpreville, S. Mussot, E. M. Flecher, and P. G. Dartevelle, "Fatal giant cell myocarditis after thymoma resection in myasthenia gravis," Journal of Thoracic and Cardiovascular Surgery, vol. 131, no. 2, pp. 494-495, 2006.

[4] N. Tanahashi, H. Sato, S. Nogawa, T. Satoh, M. Kawamura, and M. Shimoda, "A case report of giant cell myocarditis and myositis observed during the clinical course of invasive thymoma associated with myasthenia gravis," Keio Journal of Medicine, vol. 53, no. 1, pp. 30-42, 2004.

[5] M. Kodama, Y. Matsumoto, M. Fujiwara, F. Masani, T. Izumi, and A. Shibata, "A novel experimental model of giant cell myocarditis induced in rats by immunization with cardiac myosin fraction," Clinical Immunology and Immunopathology, vol. 57, no. 2, pp. 250-262, 1990.

[6] V. V. Menghini, V. Savcenko, L. J. Olson, et al., "Combined immunosuppression for the treatment of idiopathic giant cell myocarditis," Mayo Clinic Proceedings, vol. 74, no. 12, pp. 12211226, 1999. 


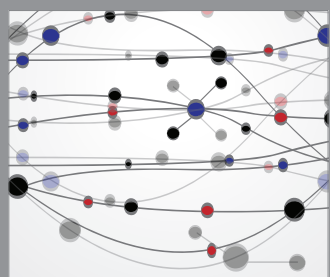

The Scientific World Journal
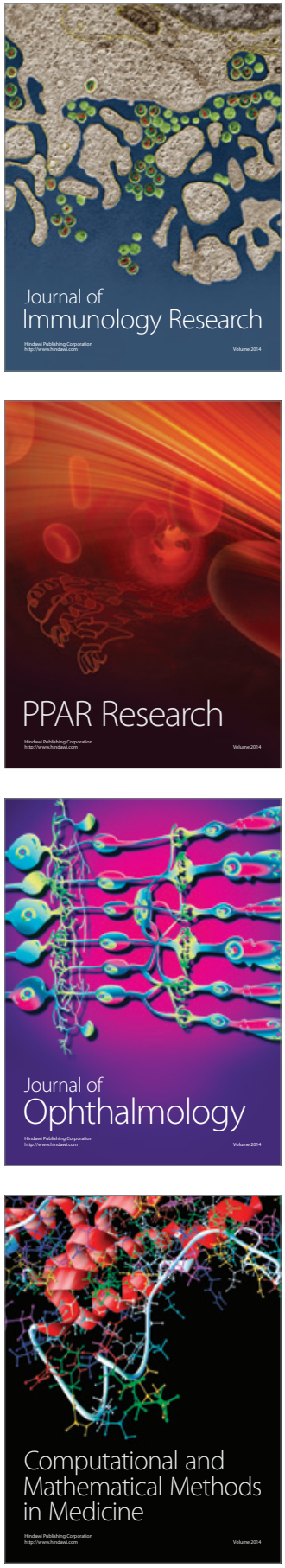

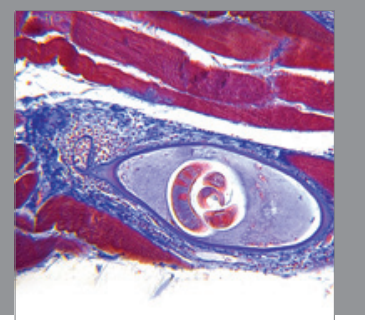

Gastroenterology

Research and Practice
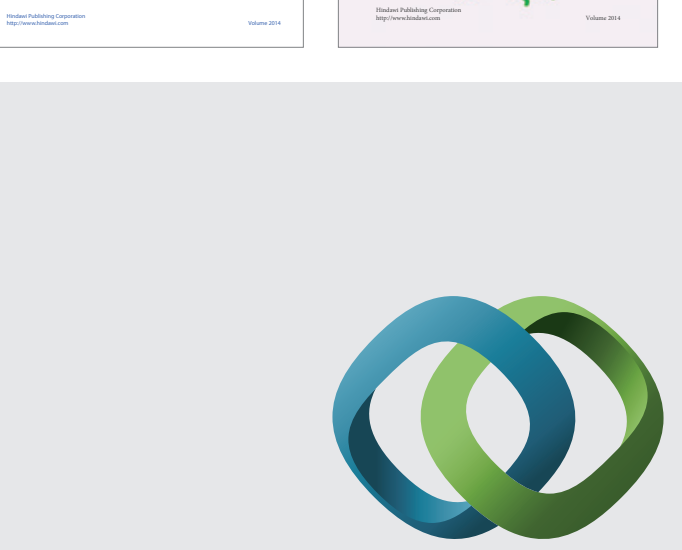

\section{Hindawi}

Submit your manuscripts at

http://www.hindawi.com
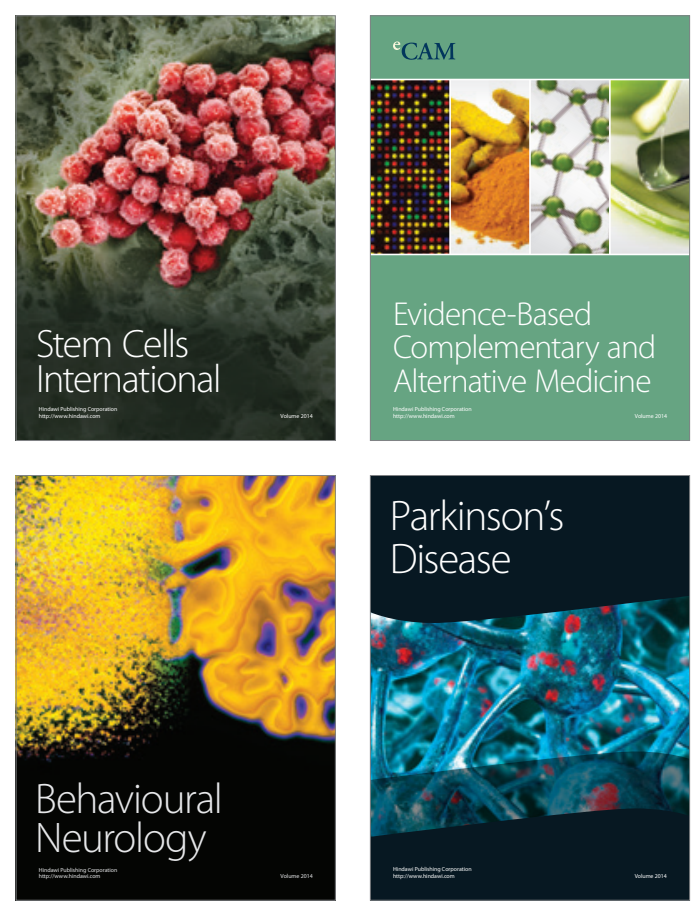

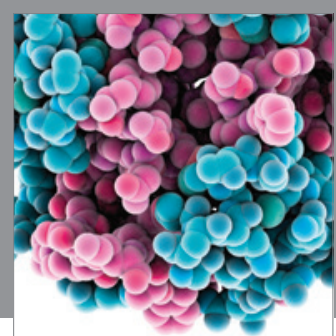

Journal of
Diabetes Research

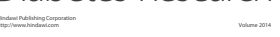

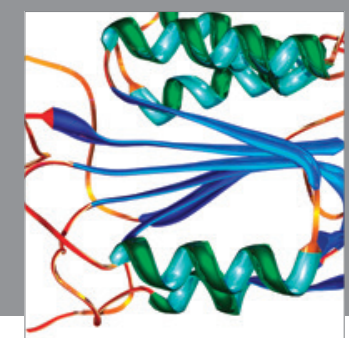

Disease Markers
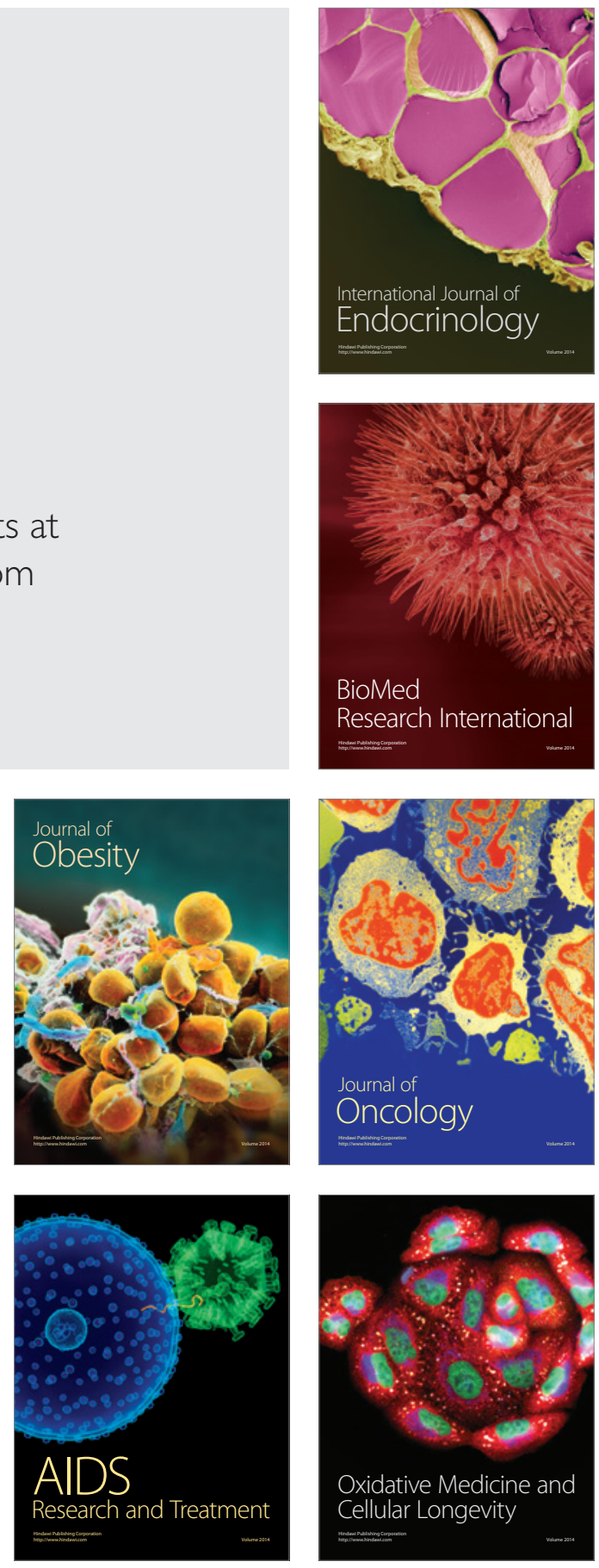